\title{
Wärmeleitfähigkeitsdetektoren-Array zur selektiven Gasanalyse
}

\author{
Stefan Dietrich, Mihails Kusnezoff, Steffen Ziesche \\ Fraunhofer IKTS Dresden, Winterbergstr. 28, 01277 Dresden \\ Jost Henze \\ Henze-Hauck Prozessmesstechnik \& Analytik GmbH, Dünnhauptstr. 14, 06847 Dessau-Rosslau
}

\section{Zusammenfassung}

Es wurde ein modularer Detektor zur selektiven Analyse von Biogas-Gemischen mit bekannten Gaskomponenten entwickelt. Das auf Wärmeleitfähigkeitsmessungen beruhende Messprinzip erlaubt die quantitative Bestimmung von maximal vier Gaskomponenten mit einer Genauigkeit von bis zu 0,3 Vol\%. Die Funktionstauglichkeit des Detektors wurde auf der Basis von Biogas-Modellzusammensetzungen demonstriert.

\begin{abstract}
A modular detector for selective analysis of biogas mixtures with known components has been developed. Measurement is based on the evaluation of thermal conductivities and allows for quantitative determination of up to four gas components with a precision of up to $0.3 \mathrm{Vol} \%$. The suitability of the sensor array has been demonstrated with different biogas compositions.
\end{abstract}

\section{Einleitung}

In vielen Bereichen der chemischen Industrie, der Biogasherstellung und der Umweltanalytik besteht die Notwendigkeit zur selektiven Analyse mehrkomponentiger Gasgemische. Der Einsatz gaschromatographischer oder massenspektrometrischer Verfahren, wie auch die Kombination verschiedener gasartspezifischer Sensoren, ist in der Regel mit erheblichen Kosten und hohem Messaufwand verbunden. Auf dem Prinzip der Wärmeleitfähigkeitsmessung beruhende Detektoren (WLD) stellen hier eine robuste und gleichzeitig kostengünstige Alternative dar. Nach bisherigem Stand der Technik erlauben diese jedoch nur die Analyse binärer Gasgemische. Eine Ausnahme stellt hier die Methode der periodischen Temperaturmodulation [1] dar, welche unter Zuhilfenahme des FTTCA-Verfahrens (Fourier-Transformed Thermal Conductivity Analysis) [2] die Analyse von Gasgemischen mit drei, in einigen Fällen auch vier, Komponenten ermöglicht. Da eine präzise Umsetzung der Temperaturmodulation jedoch mit hohen Anforderungen an Sensormaterial und Regeltechnik verbunden ist, wurde ein Messverfahren entwickelt, welches die Bestimmung der Wärmeleitfähigkeit bei unterschiedlichen, jedoch konstanten Temperaturen innerhalb eines Detektors erlaubt. Gekoppelt mit der Datenauswertung auf Basis eines Optimierungsalgorithmus ist so die selektive Analyse von Gasgemischen mit bis zu vier Komponenten möglich. Der modulare Aufbau gestattet zudem eine einfache Handhabung und erlaubt darüberhinaus den mobilen Einsatz des Detektors.

\section{Experimentelles}

\subsection{Sensoraufbau}

Grundlegende Komponenten eines Detektormoduls (Bild 1) sind bis zu vier beheizbare Sensorelemente, welche in einem thermostatisierten Gehäuse paarweise auf einen Referenz- und einen Messgas-Strang aufgeteilt und zu Wheatstone-Brücken verschaltet sind. Zur Temperierung der Sensorelemente wird jede Brückenschaltung mit einer individuellen Versorgungsspannung im Bereich von 2,5..10,0 V betrieben. Die Sensorelemente bestehen je nach Ausführung und Anforderungen aus einem keramischen oder Glas-Träger, auf welchem platinhaltige Heizelemente aufgebracht sind. Im Falle keramischer Sensorelemente werden die 
Heizer in Form von Dickschichtpasten in Siebdrucktechnik auf einem PSZ-Substrat abgeschieden und erhalten im anschließenden Sinterschritt im Hochtemperaturofen die gewünschten Eigenschaften. Sie können bei Bedarf mit weiteren Schichten zum Schutz vor unerwünschten Wechselwirkungen mit Gaskomponenten abgedeckt werden. In Bild 2 sind exemplarisch zwei keramische Sensorelemente dargestellt. Im Falle glasbasierter Sensorelemente (Bild 3) wird ein dünner Pt-Draht auf einen Quarzglaskörper gewickelt und gasdicht mit Glas eingegossen. Die einzelnen Detektormodule können mit einer unterschiedlichen Anzahl

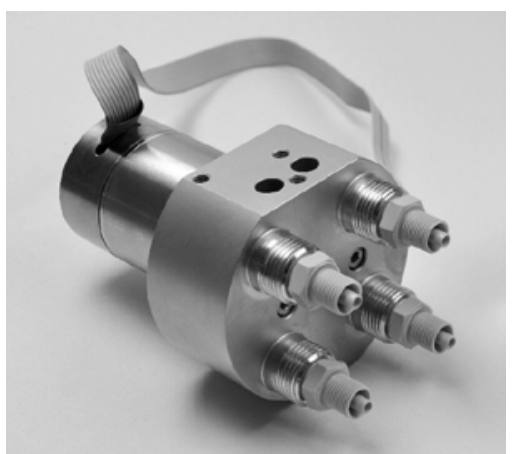

Bild 1 WLD-Detektormodul

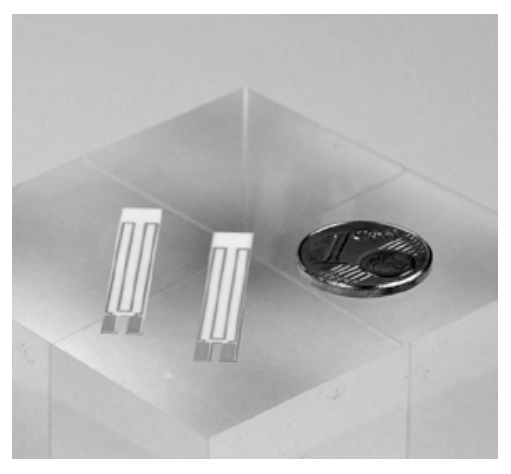

Bild 2 Keramische Sensorelemente

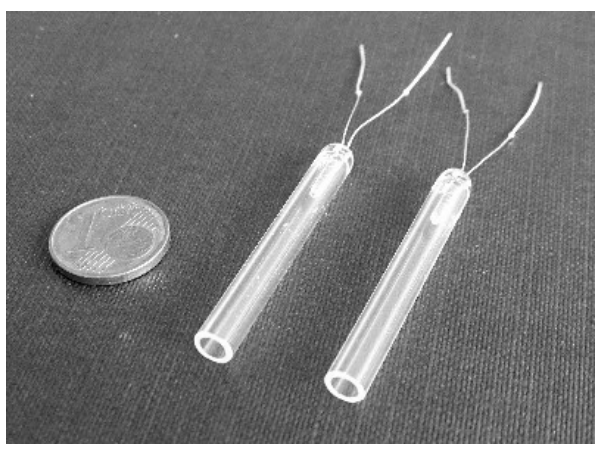

Bild 3 Glasbasierte Sensorelemente

von Sensorelementen bestückt werden und erlauben als Paar gekoppelt bei maximaler Ausbaustufe die parallele Messung bei vier unterschiedlichen Temperaturen. Sensoransteuerung sowie Datenerfassung und -auswertung erfolgen mit Hilfe eines Mikroprozessor-gesteuerten Elektronikmoduls.

\subsection{Messprinzip}

Das Messprinzip beruht auf der Ausnutzung der bekannten Temperaturabhängigkeit der Wärmeleitfähigkeit $\lambda_{k}$ einer Gaskomponente $k$,

$\lambda_{k}(T)=A_{k}+B_{k} \cdot T+C_{k} \cdot T^{2}+D_{k} \cdot T^{3}$,

$A_{k}, B_{k}, C_{k}$ und $D_{k}$ sind hierbei die tabellierten gasspezifischen Koeffizienten. Für die Wärmeleitfähigkeit eines Gasgemisches gilt nach der Mischungsregel von WASSILJEWA [3]

$\lambda_{\text {mix }}\left(\left\{X_{k}\right\}, T\right)=\sum_{k} \frac{X_{k}}{\sum_{l} \Phi_{k l}(T) \cdot X_{l}} \cdot \lambda_{k}(T)$,

wobei $X$ die entsprechenden Volumenfraktionen darstellen und die Korrekturterme $\Phi_{k l}(T)$ nach [4] gemäß

$\Phi_{k l}(T)=\left(1+\sqrt{\frac{\eta_{k}(T)}{\eta_{l}(T)} \cdot \sqrt{\frac{M_{l}}{M_{k}}}}\right)^{2} \cdot\left(\sqrt{8} \cdot \sqrt{1+\frac{M_{k}}{M_{l}}}\right)^{-1}$

berechnet werden. Hier bezeichnet $M$ die molare Masse und $\eta$ die Viskosität der entsprechenden Komponente. In der praktischen Umsetzung gelangt das in den Detektor eingeleitete Messgas durch Diffusion zum Sensorelement und bewirkt dort eine Änderung des voreingestellten Wärmeaustauschs mit dem thermostatisierten Gehäuse. Dies führt zu einer Temperaturänderung am Sensorelement und damit zu einer Änderung des elektrischen Widerstands. Die Widerstandsänderung kann nun als Variation der Brückenspannung abgegriffen und zur Auswertung an das Elektronikmodul weitergeleitet werden. Dieses übernimmt zwei Hauptaufgaben, die im Folgenden erläutert werden sollen: Die Aufbereitung der Rohmessdaten und die eigentliche Auswertung der gewonnenen Daten. 


\subsection{Datenaufbereitung und Auswertung}

Die Aufbereitung der Rohmessdaten erfolgt anhand eines detektorspezifischen Kalibrierdatensatzes, welcher mit einem geeigneten Kalibriergasgemisch erstellt wurde. In den Daten ist für die jeweilige Messtemperatur die funktionale Abhängigkeit des Spannungssignals von der tatsächlichen Wärmeleitfähigkeit über einen hinreichend großen Bereich der Gaswärmeleitfähigkeit hinterlegt. In Bild 4 sind Kalibrierkurven abgebildet, die bei vier Temperaturen mit einem variablen Gemisch aus $\mathrm{CH}_{4}$ und $\mathrm{CO}_{2}$ aufgenommen wurden. Als Ergebnis der Datenaufbereitung wird der gemessenen Brückenspannung eine

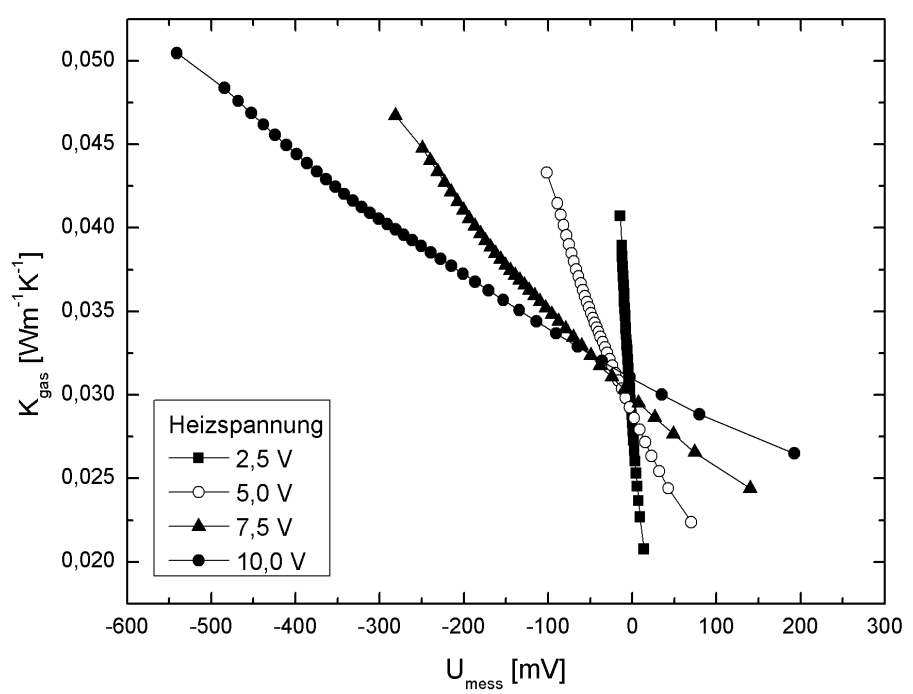

Bild 4 Kalibrierkurven für ein variables $\mathrm{CH}_{4}+\mathrm{CO}_{2}$ - Gemisch konkrete Wärmeleitfähigkeit des zu analysierenden Gasgemischs zugeordnet. Diese - je nach Anzahl der Messtemperaturen - üblicherweise drei oder vier Werte werden nun einem Optimierungsalgorithmus, dem eigentlichen Herzstück der Datenauswertung, übergeben. Der Optimierungsalgorithmus wurde auf Basis des Downhill-SimplexVerfahrens [5] in C/C\# implementiert und nutzt zur Berechnung der theoretischen Wärmeleitfähigkeiten die Mischungsregel nach Gl. 2. Neben den gemessenen Wärmeleitfähigkeiten benötigt der Algorithmus als zusätzliche Information die gasspezifischen Koeffizienten aus Gl. 1 sowie die molare Masse $M_{k}$ und die Viskosität $\eta_{k}$, welche ebenfalls als Datensatz in der Software hinterlegt sind.

Mit den vollständigen Eingangsdaten beginnt der Optimierungsalgorithmus die Suche nach der korrekten Zusammensetzung des Messgasgemischs. Als Ausgangspunkt dient hierbei eine geeignet gewählte StartGaszusammensetzung, welche die im Messgas-Gemisch enthaltenen Komponenten beinhaltet und für welche die Gesamt-Wärmeleitfähigkeiten gemäß Gl. 2 berechnet werden. Diese Werte werden mit den gemessenen verglichen: Weichen sie um mehr als einen festgelegten Betrag voneinander ab, variiert der Algorithmus im nächsten Iterationsschritt die Volumenanteile der Start-Gaszusammensetzung, bestimmt wiederum die zugehörigen Wärmeleitfähigkeiten und vergleicht diese mit den Messwerten. Diese Prozedur wird solange durchlaufen, bis die berechneten Wärmeleitfähigkeiten im Rahmen der Fehlergrenzen mit den gemessenen übereinstimmen. Die quantitative Zusammensetzung des zugehörigen theoretischen Gasgemischs entspricht dann bis auf eine vernachlässigbare Abweichung der tatsächlichen Messgas-Zusammensetzung. Eine derartige Berechnung benötigte auf dem genutzten Desktop-PC mit $2 \mathrm{GHz}$ - Singlecore-CPU und nicht optimiertem Code im Schnitt ca. 35 ms.

\section{Ergebnisse}

\subsection{Langzeitstabilität der Sensorelemente}

Zunächst wurde die Langzeitstabilität sowohl keramischer als auch glasbasierter Sensorelemente untersucht. Hierzu wurden die Proben $500 \mathrm{~h}$ bei Temperaturen bis $100{ }^{\circ} \mathrm{C}$ in Luft ausgelagert und mit einer Spannung von $5 \mathrm{~V}$ beaufschlagt. Die relative Änderung des elektrischen Widerstands nach Auslagerung ist in Tafel $\mathbf{1}$ für verschiedene Sensorelemente angegeben. Die Abweichungen liegen im Bereich der Messungenauigkeit, was die gute Langzeitstabilität beider Arten Sensorelemente belegt.

\subsection{Synthetischer Test des Auswertungsalgorithmus}

Die Genauigkeit und Leistungsfähigkeit des Algorithmus wurde anhand computergenerierter Daten verifiziert, wobei für $1 \times 10^{6}$ zufällig generierte Vier-Gas - Gemische $\left(\mathrm{CH}_{4}, \mathrm{CO}_{2}, \mathrm{~N}_{2}, \mathrm{H}_{2}\right)$ die Volumenanteile 
der Komponenten berechnet und mit den Ausgangswerten verglichen wurden. Dabei war für praxisrelevante Komponenten-Anteile $X_{k} \geq 1$ Vol.-\% der relative Fehler der berechneten Werte in 99,5 \% aller Fälle < 0,1 \%.

\subsection{Praxistest von Detektor und Auswertung}

In Tafel 2 sind die Ergebnisse erster Detektor-Tests unter Laborbedingungen dargestellt. Hierbei fand ein Detektorarray aus zwei Modulen Anwendung, welche zusammen für die Analyse von insgesamt drei Gaskomponenten ausgelegt waren. Das entsprechende Testgasgemisch wurde in Anlehnung an tatsächliche Biogas-Zusammensetzungen mit Hilfe von Massflow-Controllern (MFCs, Fa. Bronkhorst) eingestellt. Hierbei ist zu beachten, dass bereits diese MFCs laut Herstellerangaben mit einer Stellfehler von 1..2 \% behaftet sind. Als Referenzgas kam Stickstoff zum Einsatz.

Tafel 1 Langzeitstabilität verschiedener Sensorelemente

\begin{tabular}{l|c|c|c}
\hline Heizelement & $\begin{array}{c}\mathrm{R}_{0} \\
{[\mathrm{Ohm}]}\end{array}$ & $\begin{array}{c}\left|\Delta \mathrm{R}_{\mathrm{Rel}}\right| \\
{[\%]}\end{array}$ & $\begin{array}{c}\text { Messfehler } \\
{[\%]}\end{array}$ \\
\hline IKTS-1, m. Abdeckung & 14,5 & 0,7 & 1,0 \\
\hline IKTS-2, o. Abdeckung & 17,4 & 0,6 & 1,0 \\
\hline H\&H 1, geglast & 32,9 & 0,6 & 1,0 \\
\hline H\&H 2, geglast & 33,0 & 0,3 & 1,0 \\
\hline
\end{tabular}

Tafel 2 Ergebnisse des ersten Detektortests unter Laborbedingungen

\begin{tabular}{l|c|c}
\hline Gaskomponente & Vol.-\% MFC & Vol.-\% WLD \\
\hline $\mathrm{CH}_{4}$ & & \\
\hline $\mathrm{CO}_{2}$ & 40 & 40,24 \\
\hline $\mathrm{N}_{2}$ & 30 & 30,60 \\
\hline
\end{tabular}

Ein weiterer Praxistest wurde mit einem Array aus zwei Modulen bei vier Messtemperaturen mit verschiedenen Gasgemischen durchgeführt. Die Gasmischungen wurden mit Gasmischpumpen (Digamix, Fa. Wösthoff) realisiert, welche aufgrund des geringen Stellfehlers von 0,1 Vol.-\% gegenüber MFCs eine deutlich präzisere Messgas-Konfiguration zulassen. Die Messergebnisse sind in Tafel 3 abgebildet. Sie belegen die hohe Genauigkeit des Messsverfahrens selbst bei niedrigen Gaskonzentrationen.

Tafel 3 Praxistests mit variablen Gasgemischen

\begin{tabular}{c|c|c|c|c|c|c}
\hline Gemisch & $\begin{array}{c}\text { Soll-Konz. } \\
\mathrm{CO}_{2} \text { [Vol.-\%] }\end{array}$ & $\begin{array}{c}\text { Soll-Konz. } \\
\mathrm{N}_{2} \text { [Vol.-\%] }\end{array}$ & $\begin{array}{c}\text { Soll-Konz. } \\
\mathrm{H}_{2} \text { [Vol.-\%] }\end{array}$ & $\begin{array}{c}\text { WLD-Konz. } \mathrm{CO}_{2} \\
\text { [Vol.-\%] }\end{array}$ & $\begin{array}{c}\text { WLD-Konz. } \mathrm{N}_{2} \\
\text { [Vol.-\%] }\end{array}$ & $\begin{array}{c}\text { WLD-Konz. } \mathrm{H}_{2} \\
\text { [Vol.-\%] }\end{array}$ \\
\hline & & & & & & \\
\hline 1 & $50,0 \pm 0,1$ & $50,0 \pm 0,1$ & 0 & $50,3 \pm 0,2$ & $49,1 \pm 0,2$ & $0,6 \pm 0,2$ \\
\hline 2 & $49,0 \pm 0,1$ & $50,0 \pm 0,1$ & $1,0 \pm 0,1$ & $48,8 \pm 0,2$ & $49,7 \pm 0,2$ & $1,4 \pm 0,2$ \\
\hline 3 & $47,0 \pm 0,1$ & $50,0 \pm 0,1$ & $3,0 \pm 0,1$ & $47,7 \pm 0,2$ & $49,1 \pm 0,2$ & $3,2 \pm 0,2$ \\
\hline 4 & $45,0 \pm 0,1$ & $50,0 \pm 0,1$ & $5,0 \pm 0,1$ & $44,3 \pm 0,2$ & $50,9 \pm 0,2$ & $4,8 \pm 0,2$ \\
\hline
\end{tabular}

\section{Literatur}

[1] A.U. Grunewald: Modern Thermal Conductivity Technology. Emerging Technology Conference, ISA, Houston, USA, 2001.

[2] H.S. Grienauer: FTTCA - Fourier-Transformed Thermal Conductivity Analysis. Analytical Division Conference, ISA, Los Angeles, USA, 2006.

[3] VDI-Gesellschaft Verfahrenstechnik und Chemieingenieurwesen (GVC): VDI-Wärmeatlas; 6. Auflage, VDI-Verlag, Düsseldorf 1991

[4] E.A. Mason, S. C. Saxena: Phys. Fluids , Band 1, S. 361, 1958.

[5] J.A. Nelder, R. Mead: A Simplex Method for Function Minimization. Comp. J., Band 7, S. 308, 1965. 